\title{
Uso de indicadores de nível local para análise espacial da morbidade por diarreia e sua relação com as condições de vida
}

\author{
Use of local level indicators for the spatial analysis of \\ morbidity due to diarrhea and its relation with lifestyle conditions
}

Raquel Marica Cardoso Torres ${ }^{1}$

Sonia Azevedo Bittencourt ${ }^{2}$

Rosely Magalhães de Oliveira ${ }^{1}$

Alexandre San Pedro Siqueira ${ }^{1}$

Paulo Chagastelles Sabroza ${ }^{1}$

Luciano Medeiros de Toledo ${ }^{1}$

${ }^{1}$ Departamento de

Endemias Samuel Pessoa,

Escola Nacional de Saúde

Pública (ENSP), Fundação

Oswaldo Cruz (Fiocruz). R.

Leopoldo Bulhões 1480/8,

Manguinhos. 21041210

Rio de Janeiro RJ.

raquelmct@gmail.com

${ }^{2}$ Departamento de

Epidemiologia e Métodos

Quantitativos dem Saúde,

ENSP, Fiocruz.
Abstract Every child had an average of three episodes of diarrhea per year in developing countries in the twentieth century. The decrease of the number of deaths due to diarrhea in Brazil was more closely related to the use of control techniques than to changes in lifestyle conditions. This article seeks to analyze the spatial distribution of morbidity due to diarrhea among children and its relation with lifestyle conditions. This was an ecological study, with the suburbs of the city of Itaborai as units of analysis. The population studied was the number of hospitalizations of children $<5$ years for diarrhea between 2006 and 2009, available in Hospital Information Systems. The Diarrhea Hospitalization Ratio (DHR) indicator and Composite Lifestyle Quality (CLQ) indicator were established. Diarrhea still accounts for a large number of hospitalizations of children (15.5\% between 2006 and 2009). The DHR was high in this period (69.7 hospitalizations/ $1.000 \mathrm{NV}$ ). The spatial analysis identified that the suburbs with the highest DHR were, in most cases, those with the highest population agglomerations and better lifestyle conditions.

Key words Diarrhea, Hospitalization, Hospital Information Systems, Social conditions
Resumo Nos países em desenvolvimento, cada criança, apresentou em média três episódios de diarreia por ano, no século XX. No Brasil, a diminuição do número de óbitos por diarreia esteve mais associada à aplicação de técnicas de controle do que por transformações nas condições de vida. Este artigo tem por objetivo analisar a distribuição espacial da morbidade por diarreia em crianças e sua associação com condições de vida. Estudo ecológico, tendo como unidade de análise os bairros do município de Itaboraí. A população do estudo foram as crianças $<5$ anos hospitalizadas por diarreia, nos anos 2006 a 2009, disponiveis no SIH. Foram estabelecidos os indicadores Razão de Internações por Diarreia (RID) e Indicador Composto de condições de vida (ICV). As diarreias ainda representam elevada parcela das hospitalizações em crianças (15,5\% entre 2006 e 2009). A RID foi elevada no periodo (69,7 hospitalizações/ $1.000 \mathrm{NV}$ ). A análise espacial identificou que os bairros com maiores valores de RID foram, em sua maioria, aqueles com maiores aglomerações populacionais e melhores condições de vida.

Palavras-chave Diarreia, Hospitalização, Sistemas de Informação Hospitalar, Condições de vida 


\section{Introdução}

Nos países em desenvolvimento, a diarreia, manifestação comum de doenças infecciosas intestinais, ainda representa uma das principais causas de morbimortalidade em crianças menores de cinco anos de idade. No século XX, mesmo com a disponibilidade de recursos técnicos de fácil aplicabilidade, cada criança, nos países em desenvolvimento, apresentou em média três episódios de diarreia por ano ${ }^{1}$.

Embora a participação das doenças infecciosas e parasitárias tenha apresentado redução expressiva no perfil da mortalidade da população brasileira, não se observa uma tendência de redução da morbidade, evidenciado através da morbidade hospitalar. Entre os anos 1984 e 2001, este grupo de causas ainda representavam cerca de 9\% do total de internações, sendo as doenças infecciosas intestinais responsáveis pela maior parcela do conjunto de internações².

No Brasil, grande parte da redução da mortalidade por diarreia em crianças teve início a partir da década de 1960, e esteve mais associada a intervenções de saúde, como a ampla difusão da Terapia de Reidratação Oral (TRO), do que por transformações nas condições de vida ${ }^{1-7}$. No entanto, as hospitalizações por doenças diarreicas ainda permanecem como causa relevante de internação em crianças menores de cinco anos, representando a segunda principal causa no âmbito da rede hospitalar do SUS em 20018. Entre os anos 1999 e 2006 , as gastroenterites infecciosas estavam entre as três principais causas de internações por condições sensíveis à atenção básica ${ }^{9}$, nos menores de cinco anos, em todas regiões brasileiras ${ }^{10}$.

Desta forma, as doenças diarreicas ainda permanecem como um problema de saúde pública, visto que a ocorrência de hospitalizações revela a inadequação de ações de controle e/ou manutenção de seus determinantes, muito relacionados com as desigualdades de condições de vida.

No Brasil, o Sistema de Informações Hospitalares do Sistema Único de Saúde (SIH-SUS) é a única fonte de informações de morbidade hospitalar nacional, que tem origem nas Autorizações de Internação Hospitalar (AIH), destinadas ao pagamento de internações. Tal sistema apresenta uma cobertura estimada de aproximadamente $70 \%$ do total de internações do país. Devido à natureza contábil-financeira do SIH-SUS, a produção de informações ocorre de forma mais ágil que a observada nos outros sistemas ${ }^{11,12}$.

A utilização de técnicas de análise espacial para análise da distribuição de agravos tem sido bas- tante utilizada, proporcionando ferramentas importantes para vigilância e controle ${ }^{13-15}$.

Diante da diminuição da sensibilidade do indicador de mortalidade como marcador da ocorrência da diarreia em crianças menores de cinco anos, a análise das hospitalizações surge como uma alternativa para a vigilância deste agravo.

O estudo teve como objetivo analisar a distribuição espacial da morbidade por diarreia em crianças menores de cinco anos de idade por bairro e sua associação com condições de vida, utilizando o município de Itaboraí (RJ) como modelo.

\section{Métodos}

\section{Área de estudo}

O município de Itaboraí pertence à Região Metropolitana do estado do Rio de Janeiro, ocupando uma área de $424 \mathrm{Km}^{2}$, constituído por 79 bairros. Os limites municipais são: Guapimirim, Cachoeiras de Macacu, Tanguá, Maricá, São Gonçalo e Baía de Guanabara ${ }^{16}$. Entre as principais rodovias que passam pelo município, destacam-se: BR-101; RJ-104, também conhecida como Rodovia Niterói-Manilha, e dentro do perímetro urbano a Avenida vinte e dois de maio, na qual se concentra a área comercial do município. No entorno destas rodovias há grande concentração populacional, devido a facilidade de acesso a outros municípios, assim como melhor infraestrutura urbana.

Segundo os dados do censo demográfico do ano 2000, o município de Itaboraí possui apenas 22,8\% dos domicílios com abastecimento de água via rede geral, 27,8\% estão ligados à rede de esgotamento sanitário e 55,5\% estavam cobertos pelo serviço público de coleta de lixo ${ }^{17}$. Apesar dos dados demonstrarem precariedade das condições de saneamento, indicando pobreza, os dados do Ministério da Saúde demonstram que são raros os casos de morte por diarreia na população de menores de cinco anos ${ }^{18}$.

\section{Desenho de estudo}

Trata-se de um estudo ecológico, cujas unidades de análise foram os bairros do município de Itaboraí. A população do estudo foi referente às hospitalizações por diarreia de crianças menores de cinco anos residentes em Itaboraí, no período de 2006 a 2009. 
Para o cálculo do indicador de morbidade por diarreia foram utilizados dados de hospitalizações obtidos no SIH-SUS (Sistema de Informações Hospitalares do SUS) ${ }^{19}$, sendo selecionadas as internações com diagnóstico de diarreia infecciosa, referentes aos códigos A.00 a A.09 da CID10. Os dados referentes aos nascidos vivos foram extraídos do Sistema de Informações de Nascidos Vivos (SINASC) ${ }^{20}$. Foram excluídos da análise os registros de internações por diarreia em crianças menores de cinco anos (SIH-SUS) e de nascidos vivos (SINASC) que não tinha informação de bairro de residência e aquelas com bairros pertencentes aos outros municípios.

Os dados utilizados para estratificação dos bairros segundo condições de vida, foram provenientes do Censo Demográfico 2000 do IBGE $^{21}$. Para compatibilizar as informações por bairro constantes no banco de dados do SIH-SUS com as bases de dados do SINASC e IBGE, foi necessário reagrupar bairros com nomes semelhantes. Ao final as bases de dados passaram a totalizar 72 bairros ao invés dos 79 existentes no município.

O indicador utilizado no estudo para análise da aglomeração populacional, denominado Proporção de Área Ocupada, foi estabelecido a partir da interpretação visual de imagens de satélite.

\section{Descrição dos indicadores}

\section{Razão de Internações por Diarreia}

Para analisar a morbidade por diarreia, foi estabelecido o indicador denominado Razão de Internações por Diarreia para os menores de 5 anos (RID), que consistiu na divisão do número de internações por diarreia em menores de cinco anos pelo número de nascidos vivos, obtidos no SINASC $^{20}$, multiplicado por 1.000 .

Indicador composto de Condições de Vida

Neste estudo, as condições de vida foram consideradas como aquelas relacionadas com condições materiais de vida referentes à renda, escolaridade e situação de saneamento, que apontam para uma realidade determinada por uma situação social e econômica.

Para isso, foi construído um indicador composto segundo condições de vida (ICV) a partir do cálculo de índices sintéticos de condições socioeconômicas, denominado Índice de Estrutura Social (IES), e de condições sanitárias do domicílio, denominado Índice de Condições Sanitárias Locais (ICSL). A construção do indicador com- posto de condições de vida realizada neste estudo foi adaptada de outros estudos ${ }^{14,15,17}$.

O Índice de Estrutura Social (IES) foi composto pelas seguintes variáveis:

- Proporção de responsáveis por domicílios particulares permanentes com rendimento nominal mensal de até 1 salário mínimo;

- Proporção de responsáveis por domicílios particulares permanentes sem instrução ou com até 3 anos de estudo.

O Índice de Condições Sanitárias Locais (ICSL) foi composto pelas variáveis:

- Proporção de domicílios particulares permanentes com abastecimento de água da rede geral e canalização em pelo menos um cômodo;

- Proporção de domicílios particulares permanentes com banheiro ou sanitário e esgotamento sanitário via rede geral de esgoto ou pluvial;

- Proporção de domicílios particulares permanentes com lixo coletado por serviço de limpeza.

Para o cálculo final do ICV foram utilizadas as variáveis reduzidas para cada indicador, obtidas através do cálculo da subtração da média e divisão pelo desvio padrão de todas as unidades de análise. Posteriormente, estas variáveis reduzidas foram somadas segundo cada índice determinado (IES e ICLS) e, em seguida, dividido pelo número de variáveis que deram origem. Como se espera uma correlação inversa entre estes índices, foi necessário multiplicar o IES por -1. Ao final foi construído um gráfico de dispersão a partir do relacionamento entres os valores dos índices de estrutura social e condições sanitárias segundo bairros e divido em quartis para classificação das condições de vida. A partir desta divisão, foram geradas nove classes que foram reagrupadas em três.

\section{Proporção de área ocupada}

O indicador denominado proporção de área ocupada representa indiretamente a aglomeração populacional, e foi constituído mediante a interpretação visual e a vetorização de dados orbitais do satélite Alos do sensor Avnir e Prism do ano de 2008, identificando as áreas com ocupação humana. Foram consideradas áreas de ocupação humana aquelas com ocupação urbana, ou seja, foram identificadas as construções, moradias e/ou prédios, inclusive aqueles de finalidade comercial. Desta forma, foram excluídas as áreas cobertas por vegetação, hidrografia e montanhas, inclusive áreas de ocupação rural, como pastagens e plantações ${ }^{17}$.

As áreas dos bairros foram calculadas a partir do software de geoprocessamento Arc Gis 10. 
O cálculo deste indicador consistiu na relação entre área ocupada por moradias e/ou prédios e a área total do bairro, medida em quilômetros quadrados, multiplicado por 100. A classificação dos bairros segundo proporção de área ocupada considerou como pontos de corte:

- $\geq 80 \%$ : alta proporção de área ocupada,

- 50 a 79\%: média proporção de área ocupada,

- $\leq$ 49\%: baixa proporção de área ocupada.

\section{Análise estatística}

As bases cartográficas digitalizadas foram obtidas pelo projeto de Pesquisa Plano de Monitoramento Epidemiológico da Implantação do Complexo Petroquímico do Rio de Janeiro / Petrobras (COMPERJ) ${ }^{17}$, que construiu a base cartográfica do município de Itaboraí com divisão por bairro e distrito, a partir da compatibilização entre as malhas digitais fornecidas pela Secretaria de Planejamento e Coordenação do município, setor de Geoprocessamento, e a base cartográfica do IBGE $^{21}$, disponibilizada por setores censitários. Tal projeto de pesquisa tem como objetivo geral o acompanhamento analítico da evolução de doenças e agravos na área de abrangência do COMPERJ, notadamente nos municípios de Itaboraí, Guapimirim e Cachoeiras de Macacu.

Para identificar áreas de maior concentração de internações por diarreia em crianças foi utilizada a técnica de Kernel. O mapa de Kernel permite estimar a quantidade de eventos por unidade de área em cada célula de uma grade regular que recobre a região estudada ${ }^{22,23}$. Foi gerado o mapa de Kernel da RID utilizando-se a largura

Tabela 1. Número de internações em menores de cinco anos segundo diagnóstico e número de nascidos vivos segundo informação de bairro, no período de 2006 a 2009.

\begin{tabular}{lrrrrr}
\hline \multicolumn{1}{c}{ Variáveis } & 2006 & $\mathbf{2 0 0 7}$ & $\mathbf{2 0 0 8}$ & $\mathbf{2 0 0 9}$ & Total \\
\hline $\begin{array}{l}\text { Internação por } \\
\text { diarreia (n) }\end{array}$ & 277 & 191 & 220 & 230 & 918 \\
$\begin{array}{l}\text { Nascidos Vivos (n) } \\
\text { Razão de internação }\end{array}$ & 2.940 & 3.106 & 2.859 & 2.790 & 11.695 \\
por diarreia & 94,22 & 61,49 & 76,95 & 82,44 & 78,50 \\
\hline
\end{tabular}

*Foram excluídos 3,7 \% dos registros $(\mathrm{n}=34)$ : 14 por ausência de informação de bairro de residência e 20 por serem bairros de outros municípios próximos. ${ }^{* *}$ Foram excluídos $11,2 \%$ dos registros $(\mathrm{n}=1.468)$ por ausência de informação de bairro de residência: $2006(\mathrm{n}=280) ; 2007(\mathrm{n}=361) ; 2008(\mathrm{n}=445) ; 2009$ $(\mathrm{n}=382) .{ }^{* * *}$ Número de internações por diarreia em menores de cinco anos / Número nascidos vivos (x 1.000). de banda de 2.000 metros. A entrada de dados foi realizada na forma de unidades de área, neste caso específico, os bairros do município de Itaboraí, cujos centróides foram atribuídos ao atributo "RID" calculados automaticamente pelo Arc Gis 10. Os dados foram georreferenciados na projeção Sistema Universal Transverso de Mercator \% UTM, e SAD 69.

\section{Considerações éticas}

Este projeto de pesquisa foi aprovado pelo Comitê de Ética em Pesquisa da Escola Nacional de Saúde Pública Sérgio Arouca (Fiocruz).

\section{Resultados}

Do total de internações em crianças menores 5 anos no período 2006-2009, 15,5\% (918) tiveram como diagnóstico principal diarreias infecciosas, variando entre $17,8 \%(\mathrm{n}=277)$ em 2006 e $12,8 \%(\mathrm{n}=191)$ em 2007. A RID demonstrou flutuações ao longo dos anos (Tabela 1).

Dos 72 bairros do município de Itaboraí, 52 (72,2\%) apresentaram internação por diarreia em menores de cinco anos, destes, 28,8\% (15) apresentaram mais de vinte internações.

Em relação aos indicadores do ICV, os estratos de melhores condições de vida apresentaram as médias mais elevadas dos indicadores de saneamento (Tabela 2).

Ao analisar os bairros segundo classificação do ICV, verificaram-se valores de RID semelhantes entre os estratos de condições de vida baixas $(58,7)$ e altas $(57,5)$, porém, os bairros com melhores condições de vida (ICV = alto) foram os que apresentaram maior número absoluto de internações $(\mathrm{n}=514)$ (Tabela 2$)$.

O mapa de kernel da RID apresentou maior densidade nos bairros da região central, no entorno das principais rodovias. Estes bairros foram, em sua maioria, aqueles com as melhores condições de vida (ICV = alto). Também se destacaram com relevantes densidades de RID, alguns bairros mais afastados da região central (Figura 1).

Os bairros com maior densidade de RID também foram os que apresentaram maiores proporções de área ocupada. As menores densidades de RID estão nos bairros que apresentaram baixa aglomeração população, com baixos valores de proporção de área ocupada, em sua maioria bairros de características rurais, apesar desta relação não ter sido testada (Figura 2). 
Tabela 2. Proporção das variáveis por bairro que compuseram o ICV segundo estrato de condições de vida.

Média Desvio-padrão Mediana Mínimo Máximo

\section{ICV Alto ( $\mathrm{N}=25$ bairros)}

Proporção de responsáveis por domicílios (Média \%)

Rendimento $\leq 1$ salário

Sem instrução ou com até 3 anos de estudo

Proporção de domicílios (Média \%)

Abastecimento de água via rede geral

Esgotamento via rede geral ou pluvial

Lixo coletado por serviço de limpeza

Razão de internação por diarreia (por $\left.1.000 \mathrm{NV}^{*}\right)^{* *}$

ICV Médio ( $\mathrm{N}=21$ bairros)

Proporção de responsáveis por domicílios (Média \%)

Rendimento $\leq 1$ salário mínimo

Sem instrução ou com até 3 anos de estudo

Proporção de domicílios (Média \%)

Abastecimento de água via rede geral

Esgotamento via rede geral ou pluvial

18,08

29,18

3,74

6,19

32,12

40,44

28,69

18,94

75,32

17,74

57,50

39,58

23,98

37,53

3,76

4,75

24,14

38,15

17,12

28,75

29,84

20,21

17,99

16,98

11,48

32,58

19,23

16,02

18,11

0,00

0,35

6,39

$23,86 \quad 0,00$

23,99

40,88

Razão de internação por diarreia (por $1.000 \mathrm{NV})^{* * *}$

ICV baixo ( $\mathrm{N}=26$ bairros)

Proporção de responsáveis por domicílios (Média \%)

Rendimento $\leq 1$ salário mínimo

Sem instrução ou com até 3 anos de estudo

Proporção de domicílios (Média \%)

Abastecimento de água via rede geral

Esgotamento via rede geral ou pluvial

Lixo coletado por serviço de limpeza

Razão de internação por diarreia (por $1.000 \mathrm{NV})^{+* * *}$

$$
\begin{array}{r}
30 \\
46 \\
9 \\
4 \\
12 \\
58
\end{array}
$$

$\begin{array}{rrrrr}30,58 & 4,86 & 30,00 & 22,83 & 40,61 \\ 46,56 & 5,88 & 47,26 & 35,89 & 58,60 \\ & & & & \\ 9,53 & 15,44 & 0,77 & 0,00 & 55,79 \\ 4,03 & 5,00 & 2,20 & 0,00 & 20,31 \\ 12,19 & 16,97 & 6,47 & 0,00 & 82,29 \\ 58,69 & 52,18 & 64,52 & 0,00 & 176,47\end{array}$

${ }^{*} \mathrm{NV}=$ Nascidos Vivos. ${ }^{* *}$ Número absoluto de internações $=514 .{ }^{* * *}$ Número absoluto de internações $=171 .{ }^{* * * *}$ Número absoluto de internações $=199$.

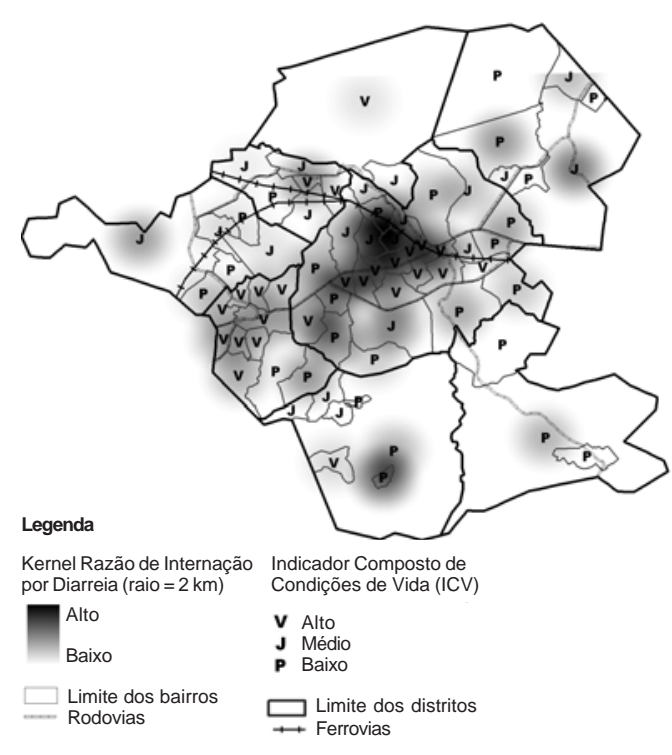

Figura 1. Mapa de Kernel da Razão de Internação por Diarreia em crianças menores de 5 anos (RID) (2006-2009) e classificação segundo ICV (2000).

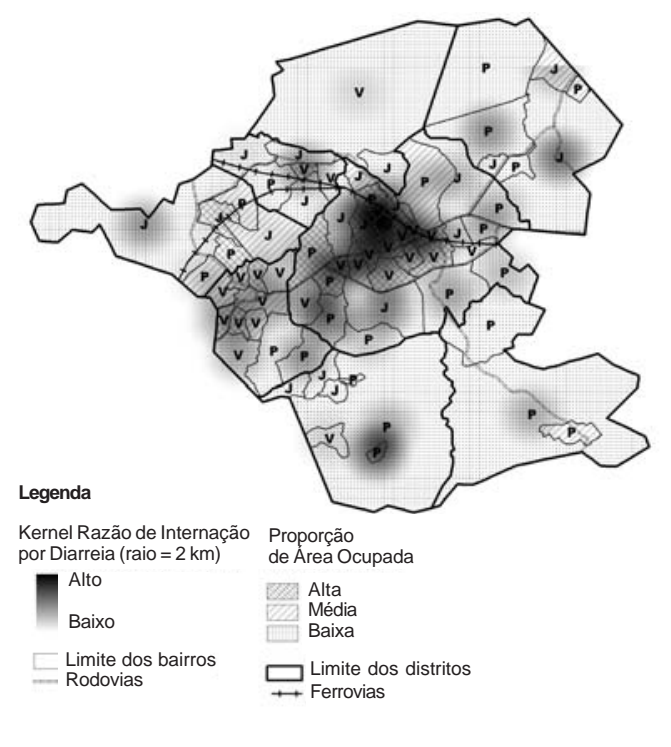

Figura 2. Mapa de Kernel da Razão de Internação por Diarreia em crianças menores de 5 anos (RID) (2006-2009) e proporção de área ocupada por bairro. 
Em relação aos períodos (2006-2007; 20082009) observou-se que houve aumento da RID entre os bairros com médias e baixas condições de vida em todos os estratos de proporção de ocupação (alta, média e baixa). Padrão diferente foi observado para aqueles que apresentaram altas condições de vida, os quais houve um decréscimo da RID de um período para o outro (Gráfico 1).

\section{Discussão}

O presente estudo identificou que as diarreias ainda representam elevada parcela das hospitalizações em menores de cinco anos (15,5\% entre 2006 e 2009), no município de Itaboraí. A Razão de Internação por Diarreia (RID) também foi elevada apresentando em média 69,7 hospitalizações por 1.000 nascidos vivos, no período. A maioria, $72,2 \%(\mathrm{n}=52)$ dos bairros do município de Itaboraí apresentou hospitalizações por diarreia, sendo que $28,8 \%(n=15)$ apresentaram mais de vinte internações e destes, 10 bairros apresentaram entre 40 e 82 hospitalizações.

Para compreensão das relações entre saúde, condições socioeconômicas e ambientais foi utilizada como metodologia a estratificação dos bairros do município de Itaboraí segundo classificação do Indicador Composto de Condições de Vida (ICV). O indicador composto é uma medida que associa diferentes variáveis num indicador sintético para analisar as características de grupos populacionais vivendo em determinadas áreas geográficas e que reflete as circunstâncias materiais ou sociais da área em questão ${ }^{24}$. Desta forma, os índices sintéticos de condições de vida têm sido apontados como opção para superar a concepção de pobreza unicamente como insuficiência de renda ${ }^{25,26}$.

Os bairros do município de Itaboraí apresentaram precárias condições de saneamento dos domicílios. Embora aqueles com melhores condições de vida tenham apresentado as médias mais elevadas dos indicadores de saneamento, esses valores ainda são distantes de uma situação ideal. Os bairros com ICV alto apresentaram 32,1\% dos domicílios com abastecimento de água via rede geral, 40,4\% dos domicílios com esgotamento sanitário via rede geral ou pluvial e $75,3 \%$ com coleta de lixo por serviço de limpeza. É importante destacar que o ICV permitiu apenas verificar a disponibilidade de infraestrutura de saneamento por meio de uma política pública, mas não permitiu verificar a qualidade. Os dados secundários do censo (IBGE) consideram apenas número de ligações para abastecimento de água e esgotamento. Porém, o abastecimento efetivo de água destes domicílios também depende de características de projeto e operação, tais como a pressão, vazão, regime de fluxo da rede e frequência, bem como a disposição desta população de pagar por este serviço ${ }^{27,28}$.

Apesar das precárias condições de vida, o município de Itaboraí não apresentou casos de

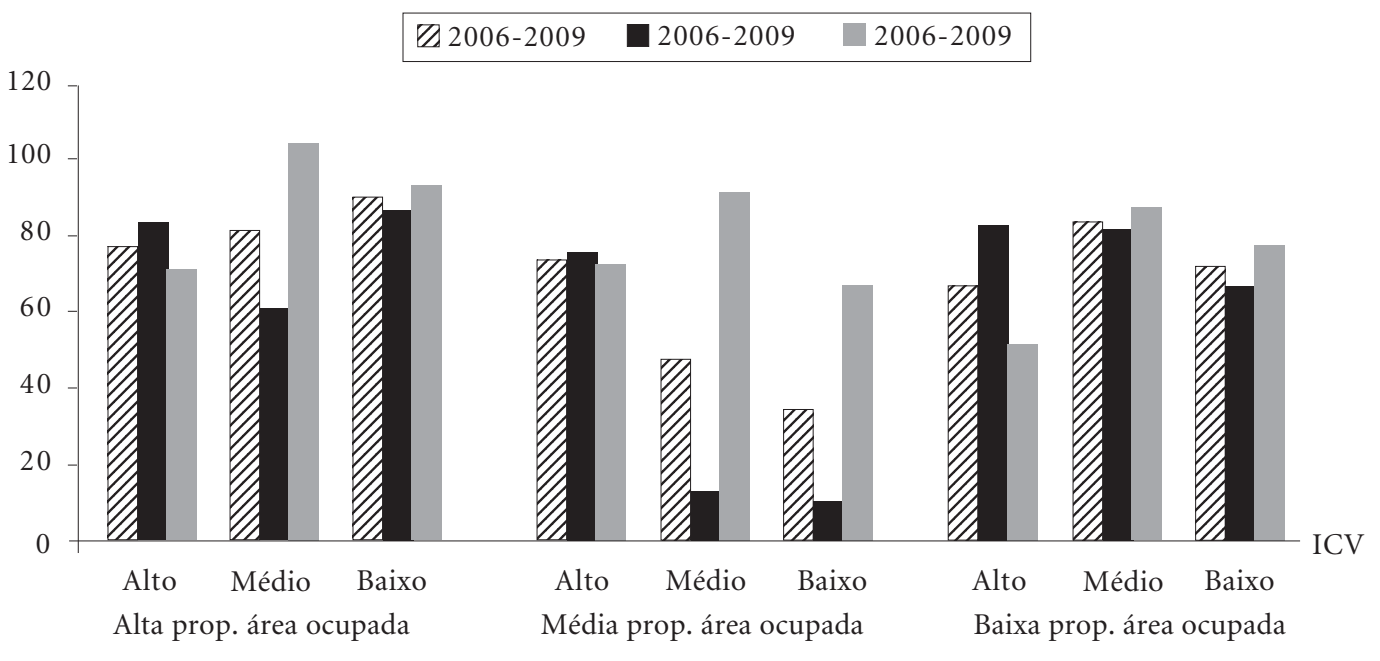

Gráfico 1. Razão de Internação por diarréia (RID) em menores de 5 anos segundo proporção de área ocupada, ICV e período de hospitalização (2006-2009). 
mortalidade por diarreia. Cujo número vem variando entre zero e dois, desde $1997^{18}$. A redução da mortalidade por diarreia pode indicar um adequado manejo através de ações de Atenção Básica de Saúde. Em décadas atrás, as medidas de Atenção Básica foram essenciais na diminuição do óbito infantil, principalmente por doenças infecciosas, como diarreias e pneumonias $^{3,4,29,30}$. Entretanto, a morbidade por diarreia em crianças menores de cinco anos ainda é relevante, evidenciada através das internações. Tal fato demonstra a perda de sensibilidade do indicador de mortalidade para análise da morbidade por diarreia em crianças.

As razões para a manutenção da ocorrência dos episódios de diarreia residem, em grande parte, na persistência dos seus fatores determinantes, externos às ações típicas do setor Saúde: urbanização acelerada sem adequada infraestrutura urbana, baixas condições de vida, condições de habitação insatisfatória principalmente em relação ao saneamento básico, alterações do meio ambiente. Também pode estar relacionada à cobertura inadequada e de baixa resolutividade dos serviços de saúde no nível primário ${ }^{1,2,10,31}$.

A análise espacial, através da estimativa de Kernel, permitiu identificar alguns padrões. Os bairros da região central, que ficam no entorno das principais rodovias, BR-101, RJ-104 e Av. vinte e dois de maio, foram os que apresentaram as maiores concentrações populacionais, assim como melhores condições de vida, ou seja, melhor infraestrutura e renda, e também concentraram os maiores valores da razão de internação por diarreia (RID) em crianças. Tal fato aponta para uma relação entre as internações por diarreia e aglomeração/dispersão populacional. Também é importante destacar que a região central concentra a maioria dos hospitais do município, e assim, esta população teria mais facilidade de acesso à internação hospitalar.

O resultado contraditório observado nos bairros do estrato de condições de vida altas que apresentaram os maiores valores de RID remete a algumas reflexões acerca dos fatores que poderiam ter contribuído para estes resultados. Uma possível explicação é a existência de problemas na constituição do próprio ICV quer em razão das variáveis escolhidas, quer por dificuldades na definição dos limites no gráfico de dispersão dos estratos, ou por heterogeneidade intra e interbairros. Outra hipótese pode estar relacionada a erros de preenchimento do endereço de residência do paciente, que muitas vezes, coloca como endereço bairros próximos do hospital como garantia de conseguir ser atendido neste estabelecimento de saúde.

Embora o estudo tenha utilizado um indicador composto como metodologia para análise das condições de vida, esta pode não ter sido a melhor opção, visto que o ICV apresentou elevada heterogeneidade entre as variáveis que o compuseram, verificado através dos elevados valores de desvio padrão e amplitude entre os valores mínimos e máximos.

A análise gráfica dos bairros de acordo com a classificação de proporção de área ocupada demonstrou que entre os bairros com maior aglomeração populacional, os maiores valores de RID estavam naqueles com piores condições de vida. Além disso, independente da aglomeração populacional, os bairros com melhores condições de vida tiveram redução da RID entre os períodos 2006-2007 e 2008-2009, enquanto os bairros com ICV médio e baixo aumentaram. Tal relação corrobora com a associação classicamente esperada, no qual as piores condições de vida estão relacionadas com maior número de casos de diarreia ${ }^{32-34}$.

O rápido processo de expansão urbana das cidades brasileiras, o adensamento populacional e a ocupação de áreas periurbanas e rurais, resultou em deficiências no saneamento básico. O suprimento de água para satisfazer às necessidades básicas diárias, envolve além da implantação de sistemas de saneamento, o seu funcionamento pleno e duradouro, ou seja, deve-se considerar a qualidade e a frequência dos serviços prestados à população ${ }^{27,28,35-37}$.

A aquisição de dados populacionais em períodos intercensitários é um desafio para análise de problemas de saúde e construção de indicadores. Especial atenção deve se tomar em relação a estimativas populacionais do município Itaboraí, alvo de grandes transformações, principalmente a partir de 2006 pela influência da instalação do COMPERJ. Portanto, na construção do indicador Razão de Internação por Diarreia (RID), optou-se por utilizar os dados de nascidos vivos disponibilizados anualmente ao invés da estimativa populacional para a população de menores de cinco anos.

Entre as principais limitações dos dados do SIH estão questões referentes a não distinção das reinternações; possibilidade de fraudes pela natureza contábil do sistema; a não universalidade, por incluir apenas as internações da rede hospitalar do SUS e as doenças com maior probabilidade de internar e deficiências em matéria de equidade e acesso aos serviços ${ }^{38-42}$. Porém, o município de 
Itaboraí possui apenas 9,8\% da população utilizando planos de saúde privados ${ }^{43}$. Em relação às reinternações, que podem gerar viés nas estimativas, foi descrito por outro estudo, em 1996, no Estado do Rio de Janeiro, no qual foi constatado que apenas 3,9\% eram de reinternações, do total de internações no SIH-SUS por doenças diarreicas infecciosas em menores de um ano ${ }^{40}$.

Embora não tenha sido objetivo deste estudo avaliar a qualidade dos sistemas de informação, é importante destacar que o preenchimento incorreto ou a incompletude das variáveis de endereço dificultaram a análise espacial. A análise dos dados do SIH-SUS identificou que não existe uma padronização no preenchimento das variáveis sobre endereço de residência, variando entre os anos analisados.

A construção do indicador de condições de vida foi baseado nos dados no censo do IBGE realizado em 2000, o que certamente pode influenciar os resultados. Porém, espera-se que as condições de vida tenham melhorado e, assim, os resultados provavelmente seriam mantidos, nos quais a RID foi mais elevada nos bairros com ICV alto.

A análise de informações geradas a partir de dados secundários sempre requer cautela. No entanto, apesar das limitações, trabalhar com as informações oficiais dos sistemas permite contribuir na discussão sobre a gestão dos dados e no processo de sua melhoria.

Os resultados demonstraram que a diarreia em crianças ainda permanece como problema relevante de saúde pública, no entanto ainda carecem de indicadores para vigilância e monitoramento deste agravo. Neste sentido, a proposta de utilização de um indicador de morbidade por diarreia a partir de dados de internação hospitalar de fácil acesso é essencial para a realização da vigilância deste agravo. Apesar do indicador utilizado neste estudo, ter subestimado a magnitude da ocorrência de diarreia porque somente as formas clínicas mais graves necessitam de hospitalizações, esta análise permite uma aproximação do problema. Tal fato indica que a magnitude da ocorrência de diarreia em crianças é maior do que a observada neste estudo através das hospitalizações.

Do ponto de vista epidemiológico, a utilização da AIH pode não só subsidiar o monitoramento da assistência prestada às doenças diarreicas, mas constituir um poderoso instrumento da Vigilância Epidemiológica, detectando problemas a serem investigados e informando sobre a circulação de agentes etiológicos.

Construir indicadores de base territorial local é um desafio na análise de situação de saúde, uma vez que os sistemas de informação disponíveis ainda carecem de padronização no preenchimento das variáveis relacionadas ao endereço de residência, o que torna a análise desses dados mais difícil e lenta. Portanto, a análise das internações por diarreia a partir da utilização do bairro como unidade de análise é inovador e permite uma maior aproximação da realidade local.

A utilização do bairro como unidade da análise espacial explicita as diversas realidades que compõem o município, merecendo abordagens distintas, diferente do que se tem visto, onde as propostas de ação podem diferenciar-se segundo os municípios, mas uniformes dentro deles.

A análise espacial das internações por diarreia contribuiu para identificação de áreas para ações de vigilância e controle. No entanto, para que estas ações sejam realizadas no tempo oportuno, maiores esforços devem ser realizados para padronização do preenchimento da AIH em relação às avariáveis de endereço de residência dos pacientes.

O indicador, razão de internação por diarreia (RID), se mostrou ser um bom proxy para vigilância e monitoramento da diarreia em crianças e a análise espacial das hospitalizações também se mostrou relevante para identificar as áreas de ocorrência desses casos.

\section{Colaboradores}

RMC Torres participou de todas as etapas do estudo desenvolvendo revisão bibliográfica, coleta, processamento e análise dos dados, elaboração do artigo e redação final. SA Bittencourt e RM Oliveira foram responsáveis pela orientação geral do estudo, participando na definição do desenho metodológico, análise dos dados, discussão e revisão final da redação do artigo. A San Pedro participou da definição do desenho metodológico, análise e discussão dos dados. PC Sabroza e LM Toledo participaram da análise e discussão dos dados. 


\section{Agradecimentos}

Aos profissionais de saúde da Secretaria Municipal de Itaboraí e aos pesquisadores do projeto Plano de Monitoramento Epidemiológico da Implantação do Complexo Petroquímico do Rio de Janeiro / Petrobras (COMPERJ). Aos integrantes do Departamento de Endemias Samuel Pessoa, Escola Nacional de Saúde Pública Sergio Arouca, Fundação Oswaldo Cruz que de forma direta ou indireta contribuíram para realização deste trabalho.

\section{Referências}

1. Kosek M, Bern C, Guerrant RL. The global burden of diarrhoeal disease, as estimated from studies published between 1992 and 2000. Bull World Health Organ 2003; 81(3):157-234.

2. Carmo EH, Barreto ML, Silva Júnior JB. Mudanças nos padrões de morbimortalidade da população brasileira: os desafios para um novo século. Epidemiol Serv Saúde 2003; 12(2):63-75.

3. Szwarcwald CL, Chequer P, Castilho EA. Tendências da mortalidade infantil no Brasil nos anos 80. Inf Epidemiol SUS 1992; 1:35-50.

4. Monteiro CA, Iunes RF, Torres AM. A evolução do país e de suas doenças: síntese, hipóteses e implicações. In: Monteiro CA, organizador. Velhos e novos males da saúde no Brasil: a evolução do país e suas doenças. São Paulo: Hucitec; 1995. p. 349-356.

5. Ometto AMH, Furtuoso COM, Silva MV. Economia brasileira na década de oitenta e seu reflexos nas condições de vida da população. Rev Saude Publica 1995; 29(5):403-414.

6. Campos GJV, Reis Filho AS, Silva AAM, Novochadlo MAS, Silva RAS, Galvão CES. Morbimortalidade infantil por diarréia aguda em área metropolitana da região nordeste do Brasil, 1986-1989. Rev Saude Publica 1995; 29(2):132-139.

7. César JA, Victora CG, Barros FC, Ramos FA, Albernaz EP, Oliveira LM, Halpern R, Breitenbach A, Stone $\mathrm{MH}$, Francalossi V. Hospitalizações em menores de um ano pertencentes a duas coortes de base populacional no Sul do Brasil: Tendências e diferenciais. Cad Saude Publica 1996; 12(Supl. 1):67-71.

8. Brasil. Ministério da Saúde (MS). Organização Mundial da Saúde, Organização Pan-Americana da Saúde. Atenção integrada às doenças prevalentes na infância. 2a Edição. Brasília: MS; 2003.

9. Brasil. Ministério da Saúde (MS). Publica em forma do anexo a Lista Brasileira de Internações por Condições Sensíveis à Atenção Primária. Portaria n. 221, 17 abril 2008. Diário Oficial da União 2007; 21 set.

10. Moura BLA, Cunha RC, Aquino R, Medina MG, Mota ELA, Macinko J, Dourado I. Principais causas de internação por condições sensíveis à atenção primária no Brasil: uma análise por faixa etária e região. Rev Bras Saude Mater Infant 2010; 10(Supl. 1):S83-S91.

11. Levcovitz E, Pereira TRC. SIH/SUS (Sistema AIH): uma análise do sistema público de remuneração de internações hospitalares no Brasil-1983-1991. Rio de Janeiro: UERJ, IMS; 1993. (Estudos em saúde coletiva, 57).

12. Carvalho DM. Grandes Sistemas Nacionais de Informação em Saúde: Revisão e Discussão da Situação Atual. Inf Epidemiol SUS 1997; 6(4):7-46.

13. Chiesa AM, Westphal MF, Akerman M. Doenças respiratórias agudas: um estudo das desigualdades em saúde. Cad Saude Publica 2008; 24(1):55-69.

14. Machado JP, Oliveira RM, Souza-Santos R. Análise espacial da ocorrência de dengue e condições de vida na cidade de Nova Iguaçu, Estado do Rio de Janeiro, Brasil. Cad Saude Publica 2009; 25(5):1025-1034.

15. San Pedro A, Souza-Santos R, Sabroza PC, Oliveira RM. Condições particulares de produção e reprodução da dengue em nível local: estudo de Itaipu, Região Oceânica de Niterói, Rio de Janeiro, Brasil. Cad Saude Publica 2009; 25(9):1937-1946. 
16. Toledo LM, Sabroza PC. Acompanhamento analítico da evolução de doenças e agravos segundo parâmetros epidemiológicos pré-estabelecidos (Relatório Técnico-Executivo I). In: Toledo LM, Sabroza PC, coordenadores. Plano de Monitoramento Epidemiológico do Processo de Implantação do COMPERJ [projeto de pesquisa]. Rio de Janeiro: Fiocruz; 2009.

17. Toledo LM, Sabroza PC, coordenadores. Plano de Monitoramento Epidemiológico do Processo de Implantação do COMPERJ [projeto de pesquisa]. Rio de Janeiro: Fiocruz; 2008.

18. Torres RMC. Hospitalizações por diarréia infecciosa em crianças, condições de vida e Atenção Básica à saúde no município de Itaboraí [dissertação]. Rio de Janeiro: Escola Nacional de Saúde Pública Sergio Arouca; 2011.

19. Brasil. Ministério da Saúde (MS). Sistema de Informações Hospitalares do SUS. [acessado 2013 mar 28]. Disponível em: http://www2.datasus.gov.br/ DATASUS/index.php?area $=0405$

20. Brasil. Ministério da Saúde (MS). Sistema de Informações de Nascidos Vivos (SINASC). [acessado 2013 mar 28]. Disponível em: http://portalsaude.saude. gov.br/portalsaude/texto/1757/458/sistemas-devigilancia.html

21. Instituto Brasileiro de Geografia e Estatística (IBGE). Censo Demográfico 2000. [acessado 2013 mar 28]. Disponível em: http://www.ibge.gov.br/home/estatistica/populacao/censo2000/default.shtm

22. Bailey TC, Gatrell AC. Interactive spatial data analysis. Harlow: Longman; 1995.

23. Souza-Santos R, Carvalho MS. Análise da distribuição espacial de larvas de Aedes aegypti na Ilha do Governador, Rio de Janeiro, Brasil. Cad Saude Publica 2000; 16(1):31-42.

24. Akerman M, Campanário P, Maia PB. Saúde e meio ambiente: análise de diferenciais intra-urbanos, Município de São Paulo, Brasil. Rev Saude Publica 1996; 30(4):372-382.

25. Akerman M. Examinando elementos que possam influenciar a formulação de políticas em estudos que utilizaram indicadores compostos: o chão contra o cifrão. Cien Saude Colet 2000; 5(1):115-123.

26. Costa MCN, Mota ELA, Paim JS, Silva LMV, Teixeira MG, Mendes CMC. Mortalidade infantil no Brasil em períodos recentes de crise econômica. Rev Saude Publica 2003; 37(6):699-706.

27. Barcellos C, Coutinho K, Pina MF, Magalhães MMAF, Paola JCMD, Santos SM. Inter-relacionamento de dados ambientais e de saúde: análise de risco à saúde aplicada ao abastecimento de água no Rio de Janeiro utilizando Sistemas de Informações Geográficas. Cad Saude Publica 1998; 14(3):597-605.

28. Borja PC, Morales LRS. Indicadores de saúde ambiental com enfoque para a área de Saneamento, parte 1: aspectos conceituais e metodológicos. Eng Sanit Ambient 2003; 8(1):13-25.

29. Macinko J, Guanais FC, Souza MFM. Evaluation of the impact of the Family Health Program on infant mortality in Brazil, 1990-2002. J Epidemiol Community Health 2006; 60(1):13-19.

30. Leal MC, Szwarcwald CL. Característica da mortalidade neonatal no Estado do Rio de Janeiro na década de 80: uma visão espaço-temporal. Rev Saude Publica 1997; 31(5):457-465.
31. Waldman EA, Barata RCB, Moraes JC, Guibu IA, Timenetsky ECD. Gastroenterites e infecções respiratórias agudas em crianças menores de 5 anos, em área da região Sudeste do Brasil, 1986-1987: II Diarréias. Rev Saude Publica 1997; 31(1):62-70.

32. Guimarães ZA, Costa MCN, Paim JS, Silva LMV. Declínio e desigualdades sociais na mortalidade infantil por diarréia. Rev Soc Bras Med Trop 2001; 34(5):473-478.

33. Barreto ML, Genser B, Strina A, Teixeira MG, Assis AMO, Rego RF, Teles CA, Prado MS, Matos SMA, Santos DN, Santos LA, Cairncross S. Effect of citywide sanitation programme on reduction in rate of childhood diarrhoea in northeast Brazil: assessment by two cohort studies. Lancet 2007; 370(9600):16221628.

34. Melli LCFL, Waldman EA. Temporal trends and inequality in under-5 mortality from diarrhea. $J$ Pediatr 2009; 85(1):21-27.

35. Souza CMN. Relação Saneamento-Saúde-Ambiente: os discursos preventivista e da promoção da saúde. Saude Soc 2007; 16(3):125-137.

36. Razzolini MTP, Günther WMR. Impactos na Saúde das Deficiências de Acesso a Água. Saude Soc 2008; $17(1): 21-32$.

37. Barcellos C. Os indicadores da pobreza e a pobreza dos indicadores: Uma abordagem geográfica das desigualdades sociais em saúde. In: Barcellos C, organizador. A geografia e o contexto dos problemas de saúde. Rio de Janeiro: Abrasco; 2008. p. 107-139.

38. Veras CMT, Martins MSA. A confiabilidade dos dados nos formulários de autorização de internação hospitalar (AIH), Rio de Janeiro, Brasil. Cad Saude Publica 1994; 10(3):339-355.

39. Schramm JMA, Szwarcwald CL. Sistema hospitalar como fonte de informações para estimar a mortalidade neonatal e a natimortalidade. Rev Saude Publica 2000; 34(3):272-279.

40. Bittencourt SA. Hospitalizações por diarréia infecciosa no Estado do Rio de Janeiro. Cad Saude Publica 2002; 18(3):747-754.

41. Bittencourt SA, Camacho LAB, Leal MC. O Sistema de Informação Hospitalar e sua aplicação na saúde coletiva. Cad Saude Publica 2006; 22(1):19-30.

42. Santos AC. Sistema de Informações Hospitalares do Sistema Único de Saúde: documentação do sistema para auxiliar o uso de suas informações. [dissertação]. Rio de Janeiro: Escola Nacional de Saúde Pública Sergio Arouca; 2009.

43. Toledo LM, Sabroza PC. Monitoramento da evolução das causas relevantes de adoecimento e morte nos municípios de Itaboraí, Guapimirim e Cachoeiras de Macacu: A situação epidemiológica em 2009 (Relatório Técnico-Executivo V). In: Toledo LM, Sabroza PC, organizadores. Plano de Monitoramento Epidemiológico do Processo de Implantação do COMPERJ [projeto de pesquisa]. Rio de Janeiro: Fiocruz; 2009.

Artigo apresentado em 21/11/2012

Aprovado em 08/12/2012

Versão final apresentada em 11/12/2012 